\title{
The Use of PROSTALAC in Two-stage Reimplantation of Septic Total Knee and Hip Arthroplasty
}

\author{
BL Wam, MBBS, EK Chee, MS Ortho, TW Ewe, FRCS \\ Department of Orthopaedics and Traumatology, Kuala Lumpur Hospital, Kuala Lumpur, Malaysia
}

\begin{abstract}
The PROSTALAC (PROSThesis Antibiotic Loaded Acrylic Cement) functional spacer is made with antibiotic-loaded acrylic cement. We use it as an interim spacer in two-stage exchange arthroplasty in cases of infected total knee or total hip replacement. PROSTALAC allows continuous rehabilitation between stages as it maintains good alignment and stability of the joint with a reasonable range of movement. It also helps to maintain the soft-tissue planes, thereby facilitating the second-stage procedure. We report here early outcomes of the use of PROSTALAC in 5 patients - 3 in total knee replacements, 1 in a total hip replacement and 1 in a bipolar hemiarthroplasty.
\end{abstract}

Key Words:

Functional Spacer, Two-stage Exchange Arthroplasty

\section{INTRODUCTION}

Despite excellent long term results after joint arthroplasty with rigorous antibiotic prophylaxis, $1.0 \%$ to $2 \%$ of the cases are complicated by infection that increases morbidity and cost. One solution following the onset of infection is the use of an antibiotic-loaded bone cement spacer after removal of the implant. In the second stage of treatment, this spacer is then replaced by another orthopaedic implant once the infection is eradicated ${ }^{3,6,10}$. Previously, pure spacers over hand-moulded implants mimicking knee or hip prostheses were used. The major disadvantages of this technique include impaired mobility, limited stability, and pain. A technique first introduced in 1987 includes the use of prefabricated articulated spacers, allowing a wider range of movement of the knee or hip ${ }^{1,2}$. The PROSTALAC (PROSThesis Antibiotic Loaded Acrylic Cement; which are made primarily of gentamicin-loaded Palacos bone cement) knee prosthesis has femoral and tibial components, however the PROSTALAC hip prosthesis has only a femoral component. Three sizes and thicknesses are available. Here, we assessed the effect of the PROSTALAC spacer on the function of the joint between stages, the eradication of infection and changes C-reactive protein (CPR) levels as a guide for re-implantation.

\section{MATERIALS AND METHODS}

We retrospectively reviewed 5 cases of infected TKR/THR (total knee replacement/total hip replacement; 3 TKR, 1 THR and 1 hemiarthroplasty) admitted to this hospital in 2009. We utilized two-stage exchange arthroplasty using the PROSTALAC prosthesis in all cases. Although PROSTALAC has a long history of use in other locales, it was only introduced in the Malaysian market recently. The mean follow up was 4 months (range, 2-8 m) after 2nd-stage of re-implantation except for Case 4.

The surgical approaches for the first stage of revision were medial parapatellar approach for knee joint and posterior approach for hip joint. After removal of the infected prosthesis and thorough debridement of all infected and necrotic tissue, the articular surfaces were prepared in a manner similar to that for revision of joint replacement without removing excessive amounts of bone. For knee joints, the PROSTALAC prosthesis was fastened with extra bone cement mixed with vancomycin. This enhances implant stability and maintains joint stability during ambulation. However, no extra bone cement was used to hold the PROSTALAC hip prosthesis; this was done to avoid difficulty in removing bone cement from the medullary canal during revision surgery. Multiple swabs were taken during the surgery to guide the choice of antibiotics after the second-stage surgery. The patella was not resurfaced.

The patients were treated on an inpatient basis with a 6 to 12 week course of intravenous antibiotics based on CRP and ESR (erythrocyte sedimentation rate) levels. Weekly ESR and CRP levels were obtained to monitor the eradication of infection and second stage revision was only carried out once ESR and CRP levels normalised. Re-implantation included the use of antibiotic-loaded (vancomycin) cement. As in the first stage, multiple swabs were taken from multiple sites during second stage surgery.

\section{RESULTS}

The patients' age ranged from 44 years old to 76 years old, with a mean age of 61.8years; the patient sample comprised 3 
Table I: Erythrocyte Sedimentary Rate (ESR)/ C-Reactive Protein(CRP) levels before and after 1st stage of surgery. All patients showed decreasing trend of ESR and CRP levels except Case 2. CRP showed early response after the 1st stage procedure compared to ESR

\begin{tabular}{|c|c|c|c|c|c|c|c|c|c|c|}
\hline & \multicolumn{2}{|c|}{ Case 1} & \multicolumn{2}{|c|}{ Case 2} & \multicolumn{2}{|c|}{ Case 3} & \multicolumn{2}{|c|}{ Case 4} & \multicolumn{2}{|c|}{ Case 5} \\
\hline & CRP & ESR & CRP & ESR & CRP & ESR & CRP & ESR & CRP & ESR \\
\hline Before ROI & 2.45 & 86 & 1.72 & 61 & 10.59 & $>120$ & 10.7 & $>120$ & 3.34 & 118 \\
\hline 1st Week & 2.00 & 74 & 2.23 & 100 & 6.1 & $>120$ & 6.01 & 110 & 7.03 & 107 \\
\hline 2nd Week & 1.72 & 46 & 1.92 & 79 & 3.39 & $>120$ & 4.05 & 85 & 1.27 & 111 \\
\hline 3rd Week & 1.03 & 30 & 2.0 & 53 & 2.1 & $>120$ & 1.77 & 60 & 0.58 & 63 \\
\hline 4th Week & 0.34 & 30 & 2.34 & 60 & & & 1.89 & 65 & 0.35 & 61 \\
\hline 5th Week & 0.30 & 24 & 2.55 & 55 & & & 0.93 & 48 & 0.30 & 48 \\
\hline 6th Week & 0.30 & 24 & 2.67 & 53 & & & 0.80 & 50 & 0.30 & 30 \\
\hline
\end{tabular}

Table II: Tissue culture and sensitivity during 1st stage operation and duration of antibiotic given

\begin{tabular}{|llll|}
\hline & Tissue C \& S & \multicolumn{1}{c|}{ Antibiotic } & Duration \\
\hline Case 1 & No growth & Ciprofloxacin and Rifampicin & 6 weeks \\
Case 2 & No growth & Ciprofloxacin and Rifampicin & 12 weeks \\
Case 3 & MSSA & Cloxacillin & 6 weeks \\
Case 4 & MSSA & Cloxacillin and fucidic acid & 6 weeks \\
Case 5 & MRSA & Vancomycin & 6 weeks \\
\hline
\end{tabular}

*MRSA = Methicillin resistant Staph. aureus, MSSA= Methicillin sensitive Staph. aureus

Table III: ESR and CRP level before 2nd stage operation, duration of 2nd stage operation after ROI and tissues $\mathrm{C}+\mathrm{S}$ taken during 2 nd stage operation

\begin{tabular}{|ccccc|}
\hline & ESR $(\mathbf{m m} / \mathbf{h r})$ & CRP $(\mathbf{m g} / \mathbf{d l})$ & $\begin{array}{c}\text { 2nd stage re-implantation } \\
\text { after ROI }\end{array}$ & $\begin{array}{c}\text { Tissue C +S during 2nd stage } \\
\text { operation }\end{array}$ \\
\hline Case 1 & 24 & 0.30 & 6 weeks & No growth \\
Case 2 & 49 & 1.17 & 6 months & No growth \\
Case 3 & - & - & Not done & No growth \\
Case 4 & 48 & 0.55 & 11 months & No growth \\
Case 5 & 30 & 0.30 & 3 months & \\
\hline
\end{tabular}

${ }^{*} \mathrm{ROI}=$ removal of implant, $\mathrm{C}+\mathrm{S}=$ culture and sensitivity

Table IV: Range of motion of affected joint after 1st-stage surgery

\begin{tabular}{|ccccc|}
\hline & Range of motion & Flexion & Extension & Abduction \\
\hline Case 1 & Hip & $100^{\circ}$ & $10^{\circ}$ & $60^{\circ}$ \\
Case 2 & Hip & $90^{\circ}$ & $15^{\circ}$ & $60^{\circ}$ \\
Case 3 & Knee & $90^{\circ}$ & $0^{\circ}$ & \\
Case 4 & Knee & $90^{\circ}$ & $0^{\circ}$ & \\
Case 5 & Knee & $75^{\circ}$ & $0^{\circ}$ & \\
\hline
\end{tabular}

men and 2 women with a mean age of 61.8 years. Two patients were diagnosed with primary osteoarthritis of the knee, 1 posttraumatic osteoarthritis of the right knee, 1 fracture neck of the left femur and 1 case of avascular necrosis (AVN) of the left hip. They presented with either knee or hip pain a few months to years after the joint replacement. ESR and CRP were high during presentation and radiography showed implant loosening (Figure 1 and Figure 2). In one case, a gallium scan was performed to confirm the diagnosis.

Intravenous antibiotics were administered for 6 weeks, after the organism was identified ${ }^{8}$ (Table II). However, two cases showed aseptic loosening (tissue culture showed no growth). These two patients were started on intravenous Ciprofloxacin and Rifampicin for 6 weeks to 3 months. The course of antibiotics was monitored through weekly CRP and ESR tests. Antibiotics were stopped when CRP and ESR normalised and re-implantation was then performed ${ }^{8,9}$. Case 2 required repeated wound debridement and an exchange of antibiotic spacer because of high CRP and ESR levels that did not show a decreasing trend (the organism was identified ${ }^{8}$ (Table I). Case 4 had delayed 2nd stage re-implantation because she developed multiple complications (upper gastrointestinal bleeding, urinary tract infection and hospital acquired pneumonia) after the 1st stage operation. Second stage reimplantation was delayed in Case 5 due to technical problems. All patients have undergone successful re-implantation except for Case 3. Case 3 had 1st stage operation only recently and is 


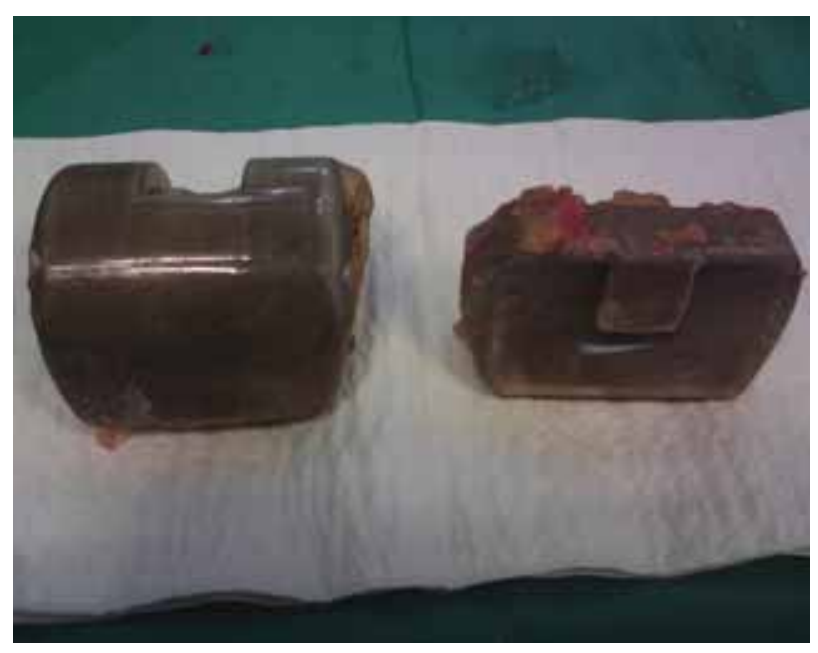

Fig. 1: PROSTALAC was removed from Case 4 during the 2nd stage of reimplantation. The surfaces of PROSTALAC were smooth and no cracks were noted after 11 months of usage.

still on intravenous antibiotics. The other 4 cases are still under follow up and do not show any signs and symptoms of infection at the time of this writing. Multiple swabs were taken during the 2 nd stage of operation and showed no organism growth.

During the intermediate phase (after 1st stage surgery), patients were ambulating with partial weight bearing. All cases were pain free with satisfactory range of motion (Table IV). Case 4 used a wheelchair but this was due to general body weakness. She was able to walk a few months after being discharged from the hospital. During this period between the two staged surgeries, no complications were seen and the wounds healed well without discharge.

\section{DISCUSSION}

There are several treatment options for infected total knee or hip replacement. In early postoperative infection $(<4$ weeks postsurgery), local debridement with primary exchange of inlay is possible. For chronic infection or after failure of other methods, a complete implant removal with arthrodesis is sometimes unavoidable. Where preservation of knee or hip function seems feasible, the two-stage exchange method with the use of acrylic bone cement spacers has gained widespread acceptance and has resulted in a high percentage of knee joints salvaged.

Antibiotics are an integral part of patient management in cases of infected TKR or THR. The choice of antibiotic prescribed in these cases is based on the culture and sensitivity obtained during the 1 st stage of surgery ${ }^{8}$. In the current series, staphylococcal species were isolated in 3 cases. This is in agreement with the work of P Dixon ${ }^{9}$ who reported that staphylococcal species were the main organisms causing infected TKRs (12 out of 15 cases). Two cases showed no microbial growth and were treated with a minimum of 6 weeks of empirical antibiotics (Ciprofloxacin and Rifampicin). This is because the bacteria most frequently encountered in chronic prosthetic infections are of the coagulase-negative Staphylococcus species. Furthermore, M Drancourt ${ }^{4}$ reported that the combination of these antibiotics achieved success rates as high as $81 \%$ for infected THRs and $69 \%$ for infected TKRs. In addition, Ciprofloxacin and Rifampicin can be taken orally leading to better compliance for long term use. The use of inflammatory markers such as ESR and CRP levelsis well established as markers of infection ${ }^{5}$ and offers a more scientific approach guiding the decision on when to end antibiotic therapy. Jason White ${ }^{5}$ agreed that CRP is a reliable indicator of early postoperative infection after TKR and THR. Thus, we used CRP and ESR levels to monitor the control of infection following the PROSTALAC insertion. All cases showed decreasing CRP and ESR levels before the 2nd stage of re-implantation took place (Table I).

For an antibiotic to be acceptable for mixing with bone cement in the operating room, it must be safe, thermo-stable, water-soluble, hypoallergenic, bactericidal with broad spectrum of activity, and in powder form. Vancomycin, tobramycin and gentamicin meet these criteria ${ }^{11}$. The use of antibiotic cement leads to high local tissue concentrations and at the same time low systemic toxicity. Not more than 6 $\mathrm{g}$ of antibiotic were added to $40 \mathrm{~g}$ of bone cement in order not to weaken the architecture of the bone cement ${ }^{5}$.

This technique has shifted over time from the use of pure spacers over hand moulded implants mimicking knee prostheses to prefabricate articulated spacers allowing a wide range of movement of the knee during the period between tstaged surgeries. PROSTALAC, an articulated gentamicinloaded polymethylmethacrylate (PMMA) spacer is a preformed articulated spacer with the biomechanical characteristics of an ultra-congruent condylar kneeprosthesis that is available in three sizes. In the present case series, $4 \mathrm{~g}$ of Vancomycin were added to the cement that holds the PROSTALAC. The rationale for this addition is because staphylococcal species are the main infectious organisms in these complications of arthroplasty.

Extensive biomechanical testing has been performed with the PROSTALAC implant; Castelli ${ }^{2}$ reported no implant breakage after in vitro testing with 500,000 cycles in dynamic mechanical testing at $1300 \mathrm{~N}$ and concluded that usage is safe for up to 6 months under strict partial weight bearing. In our series, no implant breakage occurred.

Functional outcomes for the use of PROSTALAC in this intermediate period between the first and second joint replacements are encouraging. PROSTALAC preserved limb length and positively impacted soft tissue preservation while 
at the same time allowing movement of the joint and avoiding muscular wasting. All cases were able to walk with a single cane after the 1st stage operation with the exception of Case 4, who was unable to walk due to general weakness secondary to complications developed postoperatively. Range of motion of the affected joint was satisfactory and all were able to perform daily living activities. Second-stage reimplantation was successfully carried out in 4 cases. Operation times for the second stage surgery ranged from 2$3 \mathrm{hrs}$ and were uneventful. There were no difficulties in removing the PROSTALAC. In addition, preservation of the soft tissue tension facilitated the revision surgeries.

\section{CONCLUSION}

The PROSTALAC functional spacer is useful in the twostage treatment of infected joint arthroplasty. Joint motion was maintained during the treatment period resulting in better quality of life and wider acceptance from patients. Further, 2nd-stage operation was faster and uneventful. 


\section{REFERENCES}

1. Schulz A.P, Jürgens C \& Faschingbauer M. Weight Bearing Capability of an Antibiotic Loaded Pre-Formed Articulated Knee Spacer: A Case Report. Internet J Orthop Surg 2007; 4: 1531-2968.

2. Castelli C, Martinelli R, Ferrari R. The articulated knee spacer. in: Bone Cement and cemented fixation of implants 2001; F. Pipino Ed., Zenit, Verona, Italy. 181-94.

3. Cohen JC, Hozack W, Cuckler JM, Booth RE. Two-stage reimplantation of septic total knee Arthroplasty. Report of three cases using an antibiotic-PMMA spacer block. $J$ Arthroplasty 1988; 3: 369-77.

4. Drancourt M, Stein A, J. N. Argenson, A. Zannier, Curvale G, and Raoult D.. Oral rifampin plus ofloxacin for treatment of Staphylococcus infected orthopaedic implant. Antimicrobial Agents Chemother 1993; 37: 1214-8.

5. White J, Kelly M, Dunsmuir R. C-reactive protein level after total hip and total knee replacement. J Bone Joint Surg [Br] 1998; 80(B): 909-11.

6. Masri BA, Kendall RW, Duncan CP, Beauchamp CP, McGraw RW, Bora B. Two-stage exchange arthroplasty using a functional antibiotic-loaded spacer in the treatment of the infected knee replacement: the Vancouver experience. Semin Arthroplasty 1994; 5: $122-36$.

7. McPherson EJ, Lewonowski K, Dorr LD. Techniques in Arthroplasty. Use of an articulated PMMA Spacer in the infected total knee Arthroplasty. J Arthroplasty 1995; 10: 87-9.

8. MG Wilson, K Kelley and TS Thornhill. Infection as a complication of total knee-replacement arthroplasty. Risk factors and treatment in sixty-seven cases. J Bone Joint Surg Am. 1990; 72: 878-83.

9. Dixon P,Parish E.N,Cross M.J. Arthroscopic debridement in the treatment of the infected total knee replacement. $J$ Bone Joint Surg [Br] 2004; 86(B): 39-42.

10. Scott IR, Stockley I, Getty CJ. Exchange arthroplasty for infected knee replacements: a new two stage method. J Bone Joint Surg Br 1993; 75:28-31.

11. William W. Brien, Eduardo A.Salvati, Rentaka Klein. Antibiotic impregnated bone cement in Total Hip Arthroplasty. An in vivo comparison of the evolution properties of tobramycin and vancomycin. Clin Orthop Relat Res 1993; 296: 242-8. 